\title{
Composición Química y Actividad Antibacteriana del Aceite Esencial de Minthostachys mollis
}

\author{
The chemical composition and antibacterial activity of essential \\ oil from Minthostachys mollis
}

\author{
Química Composição e Atividade Antibacteriano Óleo Essencial \\ de Minthostachys mollis
}

\author{
Miladys Torrenegra-Alarcón ${ }^{1}$, Clemente Granados-Conde ${ }^{2}$, Marlene Durán-Lengua $^{3}$, \\ Glicerio León-Méndez ${ }^{4}$, Xiomara Yáñez-Rueda ${ }^{5}$, César Martínez ${ }^{6}$, Nerlis Pájaro-Castro $^{7}$
}

1 Ing. Alim, MSc, Centro de Comercio y Servicios, Regional Bolívar. SENA. Grupo de Investigación de Biotecnología e Innovación -GIBEI-, Facultad de Ingeniería, Universidad de Cartagena. Grupo de Investigación en Ingeniería, Innovación, Calidad Alimentaria y Salud-INCAS-

2 Ing. Alim, MSc, Facultad de Ingeniería, Universidad de Cartagena. Grupo de Investigación en Ingeniería, Innovación, Calidad Alimentaria y Salud-INCAS-

3 Bact, MSc, PhD Facultad de Medicina, Universidad de Cartagena.

4 Químico Farmacéutico, MSc(C), Facultad de Ciencias Farmacéuticas, Universidad de Cartagena. Grupo de Investigación en Tecnología Farmacéutica, Cosmética y de Alimentos -GITFCA-

5 Lic. Ciencias, MSc, PhD, Facultad de Ciencias Básicas, Universidad de Pamplona. Grupo de Investigación en Productos Verdes (GPV)

6 Mic. Ind, Esp, Fundación Universitaria Tecnológico de Comfenalco.

7 QF, MSc, PhD (C) Facultad de Ciencias de la Salud, Universidad de Sucre. Grupo de Ciencias Médicas y Farmacéuticas Email: mtorrenegraa@hotmail.com

\section{Resumen}

Se evaluó la composición química y la actividad antibacteriana del aceite esencial Minthostachys mollis cultivado en el departamento de Norte de Santander, Colombia. El aceite esencial fue obtenido por hidrodestilación convencional, a partir de las hojas; se determinó densidad relativa a $20^{\circ} \mathrm{C}$, índice de refracción; solubilidad de los aceites esenciales en etanol $(70 \%$ v/v) y rotación óptica. La composición química se evaluó mediante cromatografía de gases/espectrómetro de masa. La actividad se realizó sobre tres bacterias: Staphylococcus aureus ATCC 25923, Staphylococcus epidermidis ATCC 12228 y Escherichia coli ATCC 25922. Para determinar la sensibilidad antibacteriana y la concentración mínima inhibitoria, los aceites se diluyeron hasta la concentración deseada $(1000-50 \mu \mathrm{g} / \mathrm{mL})$ empleando el método de microdilución en caldo, y se empleó el lector de microplacas para la cuantificación del crecimiento bacteriano. El rendimiento fue de 0,6\%. Los resultados de la prueba de sensibilidad mostraron que las bacterias fueron sensibles al aceite esencial de Minthostachys mollis; además, este aceite presentó un elevado contenido de monoterpenos oxigenados con reconocida actividad antibacteriana, como son el carvacrol y el timol. En función de los resultados obtenidos, concluimos que la especie vegetal evaluada es promisoria para el control del componente bacteriano.

Palabras clave: Muña, aceite esencial, actividad antibacteriana. 


\begin{abstract}
The chemical composition and antibacterial activity of essential oil from Minthostachys mollis were evaluated; the plants were grown in the Norte de Santander department, Colombia. The essential oil was obtained by conventional hydrodistiIlation from Minthostachys mollis leaves; relative density was determined at $20^{\circ} \mathrm{C}$, as were the refraction index, essential oil solubility in ethanol $(70 \% \mathrm{v} / \mathrm{v})$ and optical rotation. Chemical composition was evaluated by gas chromatography/mass spectrometry (GC-MS). Three bacteria were used for evaluating antibacterial activity: Staphylococcus aureus ATCC 25923, Staphylococcus epidermidis ATCC 12228 and Escherichia coli ATCC 25922. The oil was diluted to the desired concentration $(1,000-50 \mu \mathrm{g} / \mathrm{mL})$ for determining antibacterial sensitivity and minimum inhibitory concentration, using the broth microdilution method, and a microplate reader was used for quantifying bacterial growth. Yield was $0.6 \%$. Sensitivity test results revealed that bacteria were sensitive to essential oil from Minthostachys mollis; furthermore, this oil had a high content of oxygenated monoterpenes (i.e. carvacrol and thymol) having recognised antibacterial activity. It can thus be concluded that the vegetal species evaluated here is promising for controlling bacteria.
\end{abstract}

Key words: Muña, essential oil, antibacterial activity.

\title{
Resumo
}

Avaliou-se a composição química e atividade antibacteriana do óleo essencial Minthostachys mollis cultivada no departamento de Norte de Santander, Colômbia. O óleo essencial foi obtido por destilação convencional das folhas; determinada densidade relativa a $20^{\circ} \mathrm{C}$, índice de refração; solubilidade de óleos essenciais em álcool etílico (70\% v/v) e rotação óptica. A composição química foi avaliada utilizando o espectrômetro de massa/cromatógrafo. O evento foi realizado para três bactérias: Staphylococcus aureus ATCC 25923, Staphylococcus epidermidis ATCC 12228 e Escherichia coli ATCC 25922. Para determinar a sensibilidade antibacteriana e a concentração inibitória mínima, óleos são diluídos para a concentração desejada (1000-50 $\mu \mathrm{g} / \mathrm{mL})$, utilizando o método de microdiluição em caldo, e usado o leitor de microplacas para a quantificação do crescimento bacteriano. O rendimento foi de $0,6 \%$. Os resultados do teste de sensibilidade mostraram que as bactérias foram sensíveis para o óleo essencial de Minthostachys mollis; Além disso, este óleo apresenta um alto teor de monoterpenos, oxigenados com atividade antibacteriana conhecida, tais como o carvacrol e timol. Dependendo dos resultados, podemos concluir que as espécies de plantas avaliadas é promissora para o controle do componente bacteriano.

Palavras-chave: Muña, óleo essencial, atividade antibacteriana.

\section{Introducción}

Colombia es un país que posee una gran diversidad de ecosistemas y microclimas, lo cual hace que su vegetación sea muy variada, enriquecida con especies endémicas y diversidad genética muy alta, entre las cuales se tienen algunas que poseen aceites esenciales (AE) con principios activos con potencial actividad biológica e industrial, que convierten a nuestro país en una región muy interesante para la investigación y desarrollo de nuevos productos naturales (Granados et al., 2012; Torrenegra et al., 2015a).

Los aceites esenciales (AE) de plantas aromáticas y medicinales contienen principios activos que exhiben bioactividades como la antioxidante, antifúngica, antimicrobiana, entre otras (Matiz et al., 2012a). En particular, el AE de la especie vegetal Minthostachys mollis Griseb perteneciente al género Minthostachys conocida comúnmente como muña, es una especie de planta arbustiva leñosa, oriunda de Argentina, Bolivia, Colombia, Ecuador, Perú y Venezuela, utilizada en medicina popular para tratar los cólicos estomacales y ciertos trastornos gripales (Carhuapoma et al., 2009;Torrenegra et al., 2015a).

Adicionalmente, hoy por hoy, la tendencia de los pacientes se inclina hacia el consumo de "fitotera- péuticos", libres de productos de síntesis y aditivos químicos por lo que resulta interesante estudiar la actividad antibacteriana de los aceites esenciales (AE) de plantas nativas.

En este trabajo, se obtuvo el $\mathrm{AE}$, mediante hidrodestilación de la especie vegetal Minthostachys mollis, cultivada en el departamento de Norte de Santander (Colombia); y se determinó la composición química, la sensibilidad antibacteriana y la concentración mínima inhibitoria (CMI) in vitro del AE sobre tres bacterias: Staphylococcus epidermidis, Escherichia coli y Staphylococcus aureus.

\section{Materiales y métodos}

Las muestras de muña fueron recolectadas en el municipio de Pamplona en Norte de Santander - Colombia $\left(7^{\circ} 22^{\prime} 34^{\prime \prime} \mathrm{N} 72^{\circ} 38^{\prime} 54^{\prime \prime} \mathrm{O}\right)$. Cabe resaltar que la etapa de muestreo de la especie vegetal, se llevó a cabo durante la puesta del sol, para evitar que las altas temperaturas pudieran volatizar algunos componentes presentes en las muestras. El material vegetal se empacó en un contenedor de poliestireno expandido y se preservó a $25^{\circ} \mathrm{C}$ (Granados et al., 2012). El material vegetal fue identificado en el Herbario Regional 
Catatumbo-Sarare (HECASE) de la Universidad de Pamplona, registro nacional de colecciones biológicas. El número de colección de dicha planta fue conservado con № de colección Granados C. Nㅜ01.

\section{Procesamiento del material vegetal}

Las hojas colectadas fueron lavadas con agua y seleccionadas para garantizar buen estado; seguidamente se trocearon, pesaron y procesaron inmediatamente. La extracción del AE de las hojas se realizó por hidrodestilación convencional (HD). Se empleó un equipo de hidrodestilación con capacidad para 4 L, 500 g del material vegetal, seleccionado y troceado, se introdujeron en el balón de extracción, el cual contenía 500 $\mathrm{mL}$ de agua destilada; el tiempo de extracción fue de 3-4 horas. El AE se colectó en un recipiente tipo Dean Stark. El AE se separó por decantación e inmediatamente fue almacenado en un vial ámbar de $4 \mathrm{~mL}$ (Torrenegra et al., 2015b).

\section{Determinación de propiedades físicas del $A E$}

A cada muestra de AE se le midieron las siguientes propiedades físicas: a) densidad relativa a $20^{\circ} \mathrm{C} ; \mathrm{b}$ ) índice de refracción; c) solubilidad del $\mathrm{AE}$ en etanol $(70 \% \mathrm{v} / \mathrm{v})$ : en un tubo de plástico con tapa de $1,5 \mathrm{~mL}$ se adicionaron $100 \mu \mathrm{L}$ de etanol al $70 \%$ (v/v) y $2 \mu \mathrm{L}$ del AE. La mezcla se homogenizó en un vórtex a $200 \mathrm{rpm}$ hasta obtener una solución homogénea (Torrenegra et al., 2015b).

\section{Análisis del AE por cromatografía de gases/ espectrómetro de masa (CG/EM)}

Se empleó un equipo CG/EM 7890A/5975C Agilent (Estados Unidos) en interfase con un detector selectivo de masas HP5973 Network conectado en línea con un sistema HP-MS ChemStation y la base de datos NIST-2008. Las condiciones de operación fueron: columna capilar HP-5MS (5\% phenyl methyl silox, $30 \mathrm{~m}$ x $250 \mu \mathrm{m} \times 0,25 \mu \mathrm{m})$, temperatura inicial $45^{\circ} \mathrm{C}$, temperatura de la línea de transferencia de $280^{\circ} \mathrm{C}$ y volumen de inyección 1,0 $\mu \mathrm{L}$ en modo split (20:1), con temperatura del inyector de $250^{\circ} \mathrm{C}$. La detección de los compuestos se realizó por comparación del espectro de masas, en cada tiempo de retención, con los reportados en la base de datos NIST-2008 (Tomy et al., 1997; Baharum et al., 2010; León et al., 2015; (Torrenegra et al., 2015b).

\section{Actividad antibacteriana in vitro}

Los inóculos bacterianos se prepararon de acuerdo a las indicaciones establecidas por el Instituto de están- dares clínicos y de laboratorio (CLSI, 2011), tomando entre 3-4 colonias bien diferenciadas y morfológicamente similares de las bacterias previamente sembradas en placas Petri con el agar específico, y luego suspendiéndolas en tubos de ensayo en caldo homólogo estéril, para $S$. aureus, $S$ epidermidis y E. coli se utilizó Tripticasa Soya (TSA). La incubación se realizó a $35 \pm 2{ }^{\circ} \mathrm{C}$ y se verificó sistemáticamente la densidad óptica (DO) a $620 \mathrm{~nm}$ en lector de microplacas (Multiscan EX Thermo ${ }^{\circledR}$, Estados Unidos) hasta que la suspensión bacteriana alcanzara una DO620 entre 0,08-0,1 unidades, equivalente a 0,5 en la escala de McFarland $\left(1 \times 10^{8} \mathrm{UFC} / \mathrm{mL}\right)$; la suspensión fue diluida a fin de obtener una suspensión de trabajo de $5 \times 10^{5} \mathrm{UFC} / \mathrm{mL}$ en los ensayos biológicos. Las cepas se inocularon en el momento de mayor densidad óptica. Para ello, 0,1 mL de inóculo diluido fue adicionado a $9,9 \mathrm{~mL}$ del caldo específico, e incubado a $35 \pm 2{ }^{\circ} \mathrm{C}$ y verificando, a intervalos regulares la DO620 de la suspensión bacteriana en lector de microplacas. El tiempo en el que se logró el mayor valor, se empleó como tiempo de incubación en todos los ensayos. Debido a la insolubilidad en agua de los $\mathrm{AE}$, se utilizaron mezclas de caldo: etanol: Tween-80. Para este estudio, diferentes mezclas de etanol: caldo:tween-80 se incubaron con las cepas bacterianas en placas de 96 pocillos a $35 \pm 2{ }^{\circ} \mathrm{C}$ por el tiempo definido para cada bacteria según las curvas de crecimiento; posteriormente, se determinaron los porcentajes de viabilidad frente al blanco de máximo crecimiento (caldo con inóculo), con el objeto de seleccionar la mezcla más adecuada. Para la evaluación de la sensibilidad antibacteriana se prepararon soluciones de AE a concentración de $1000 \mu \mathrm{g} / \mathrm{mL}$, acorde con el criterio de Gibbons que considera como promisorios los productos que presenten valores de $\mathrm{CMI}$ inferior a $1000 \mu \mathrm{g} / \mathrm{mL}$. Estas soluciones se incubaron con las suspensiones bacterianas a $35 \pm 2{ }^{\circ} \mathrm{C}$, utilizando gentamicina sulfato $(0,016 \mathrm{mg} / \mathrm{mL})$ como control positivo de actividad antibacteriana. Al final del periodo de incubación, las placas se agitaron durante 5 min a 100 rpm, se determinó la DO620 en lector de microplacas y se estimó la viabilidad por comparación frente al blanco de máximo crecimiento (Gibbons 2005; Ramírez et al., 2009; Matiz et al., 2015b; (Torrenegra et al., 2015b).

\section{Concentración Mínima Inhibitoria (CMI)}

Cincuenta $\mu \mathrm{L}$ de las suspensiones de las cepas fueron incubadas en placas de 96 pozos, con $50 \mu \mathrm{L}$ de concentraciones seriadas entre 1000 y 50 partes por millón (ppm) de los aceites esenciales evaluados. Las placas fueron selladas durante la incubación para reducir la evaporación. Al finalizar, se agitaron (100 rpm, 5 
min) y se determinó la $\mathrm{DO}_{620}$ en lector de microplacas. La CMI (ppm) se calculó como la mínima concentración del aceite esencial que inhibió completamente el crecimiento, comparando contra pozos de caldo puro. Pozos con caldo inoculado (máximo crecimiento) y con gentamicina (30 ppm) se emplearon como control (Matiz et al., 2015b; (Torrenegra et al., 2015b).

\section{Análisis estadístico}

Todos los ensayos se realizaron por triplicado. Los resultados se expresaron como la media \pm DE (desviación estándar). Las diferencias significativas se determinaron mediante análisis de $t$ de student.

\section{Resultados y discusión}

La eficiencia de la extracción, las propiedades físicas del $\mathrm{AE}$ se presenta en la tabla 1.

Tabla 1. Rendimiento y propiedades físicas del AE de $M$. mollis

\begin{tabular}{|l|c|}
\hline \multicolumn{1}{|c|}{ Análisis } & M. mollis \\
\hline Rendimiento del AE & $0,6 \pm 0,05 \%$ \\
\hline Densidad especifica $20^{\circ} \mathrm{C}$ & $0,90 \pm 0,05 \mathrm{~g} / \mathrm{mL}$ \\
\hline Índice de refracción $20^{\circ} \mathrm{C}$ & $1,4774 \pm 0,02$ \\
\hline Solubilidad en etanol al $70 \%$ & Positiva \\
\hline
\end{tabular}

El AE de la especie vegetal obtenido por el método de extracción presento un olor intenso y característico, líquidos a temperatura ambiente, arrastrable por vapor de agua e insolubles en ella, coloración amarillo pálido.

Cano et al., 2008, obtuvieron un rendimiento del $0,19 \%$ por el método de arrastre con vapor de agua, muy inferior al reportado en el presente estudio $(0,6 \%)$ esto se debe principalmente a los siguientes factores: métodos de cultivo, condiciones geobotánicas: clima, altitud, tipo de suelo, luminosidad, pluviosidad, temperatura; época de recolección y edad de las plantas.

Torrenegra et al., 2015a, indicaron que a mayor índice de refracción el AE contendrá mayores terpenos bencénicos. Según este criterio el aceite esencial posee ligeramente más compuestos terpénicos.

La literatura científica menciona que valores mayores a 1,00 indican la presencia de terpenos aromáticos, nitrogenados y azufrados; en cambio, valores menores, incluso cercanos a 0,840, atestiguan la presencia de hidrocarburos aromáticos. Estos datos muestran que el aceite esencial posee mayores terpenos oxigenados (Torrenegra et al., 2015a).

La prueba de solubilidad en etanol al $70 \%$ fue positiva para los diferentes AE obtenidos ambos métodos. El comportamiento de solubilidad es semejante al reportado por Torrenegra et al., 2015a; lo anterior, se debe principalmente al contenido de compuestos oxigenados en los AE. La presencia de compuestos oxigenados aumenta la afinidad por el solvente $y$, adicionalmente, los aldehídos y alcoholes poseen la capacidad de formar puentes de hidrógeno; por tal razón, el contenido de compuestos oxigenados, además de proveer las notas aromáticas agradables a los $\mathrm{AE}$, aumentan su solubilidad en etanol haciéndolos más aptos para su aplicación en la industria.

La identificación de los componentes, los tiempos de retención y porcentajes de abundancia del AE de $M$. mollis (muña) por CG/EM son reportados en la tabla 2. El compuesto mayoritario encontrado es el carvacrol con un porcentaje de abundancia relativa de $21,24 \%$.

Tabla 2. Componentes mayoritarios detectados en el AE de M. mollis.

\begin{tabular}{|l|c|}
\hline \multicolumn{1}{|c|}{ Compuesto } & $\begin{array}{c}\text { \% Abundancia } \\
\text { relativa, (tR, min) }\end{array}$ \\
\cline { 2 - 2 } o-pineno & M. mollis \\
\hline Limoneno & $1,73(7,34)$ \\
\hline Carvacrol & $0,56(10)$ \\
\hline Eucaliptol & $21,24(10,07)$ \\
\hline Timol & $10,04(10,07)$ \\
\hline Pulegona & $13,11(13)$ \\
\hline Germacreno-D & $9,84(41)$ \\
\hline Biciclogermacreno & $11,85(44,36)$ \\
\hline Acetato de cariofileno & $1,83(44,803)$ \\
\hline Longifolol & $1,83(45,7)$ \\
\hline Acetato de $\alpha$-Eudesmol & $1,77(46,11)$ \\
\hline Acetato de (-)-Isolongifolol & $11,32(47,588)$ \\
\hline Acetato de exo-Norbornanol & $3,94(49,87)$ \\
\hline
\end{tabular}

Tiempo de retención (tr) y abundancia relativa (\%) de los aceites esenciales, identificados por comparación con espectro de masas de referencia de la base de datos NIST - 2008. 
Cano et al., 2008, encontraron como componente mayoritario del aceite esencial de Muña al pulegona (36,68\%). Resaltado que este compuesto está presente en el perfil cromatográfico mostrados en la tabla 2.

Sin embargo, es visible una pequeña variación con respecto a los componentes minoritarios encontrados por Cano et al., 2008, ocasionado por el efecto de diferentes factores ambientales sobre el contenido de compuestos en plantas medicinales. La intensidad de la luz y el fotoperíodo, que varían de región en región; época de recolección y edad de las plantas afectan la composición del aceite esencial.

Los ensayos de crecimiento revelaron que las cepas de $S$. aureus, $S$. epidermidis y E. coli alcanzaron la mayor DO620 a las 20 horas, estos fueron los tiempos de punto final de incubación en los bioensayos de actividad antibacteriana. Para solubilizar los aceites, se demostró que las mezclas que contenían etanol al 4 $\%$ y polisorbato-80 al $1 \%$ no inhibieron el crecimiento de ninguna de las cepas, por tanto, se eligió utilizar un sistema caldo: etanol: polisorbato-80 en proporción 95:4:1, para solubilizar el aceites esenciales evaluado en este trabajo.

Los resultados de la evaluación de la sensibilidad antibacteriana del AE (tabla 3), permitió escoger el aceite en estudio, tomando como criterio de selección, que fuera capaz de inhibir en más de un 90\% a las tres cepas. La cepa menos sensible a los aceites fue la $S$. epidermidis.

La CMI se determina con la utilización de caldo inoculado y estandarizado, al que se adicionan soluciones de aceites a diferentes concentraciones, provocando una dilución; esto explica el por qué la absorbancia (DO620) del caldo puro es mayor que la de los demás pozos al tiempo inicial de incubación. Al final de la misma, se leen las absorbancias de todos los pozos. Se considera inhibición total, en aquellos con valores inferiores al del caldo puro. La mayor concentración de aceite capaz de lograr esto, se denomina CMI. Los valores se presentan en la tabla 4.

Se reconoce que los $\mathrm{AE}$ dependen de sus propiedades lipofílicas o hidrofílicas. Los terpenoides pueden servir como un ejemplo de agentes liposolubles, los cuales afectan la actividad de las enzimas catalizadoras a nivel de membrana, por ej. Ciertos componentes del AE pueden actuar como desacopladores, los cuales interfieren en la translocación de protones sobre la membrana y subsecuentemente interrumpir por la fosforilación del ADP (Cano et al., 2008).

En particular, este AE presentó la mayor concentración relativa del compuesto carvacrol, el cual puede penetrar la membrana del citoplasma, causando una desestabilización de esta; igualmente podría actuar como intercambiador de protones, reduciendo el gradiente de $\mathrm{pH}$ a lo largo de la membrana (Xu et al., 2008).

De esta manera se sigue sumando evidencia sosteniendo que los $\mathrm{AE}$, son una buena fuente natural y disponible que posibilitará desarrollar diferentes formas farmacéuticas con actividad farmacológica definida. Por otro lado, estos resultados pueden servir para comenzar a entender las razones del extenso uso de los aceites esenciales, ya sean en la medicina tradicional o en la aromaterapia, al mismo tiempo podemos acercarnos cada vez más a la utilización de las plantas

Tabla 3. Porcentajes de inhibición de crecimiento obtenidos en la prueba de sensibilidad bacteriana del aceite esencial (1000 $\mu \mathrm{g} /$ $\mathrm{mL}$ ) frente a tres cepas bacterianas.

\begin{tabular}{|l|c|c|c|}
\hline \multirow{2}{*}{ Aceite esencial } & \multicolumn{3}{|c|}{ Cepas bacterianas } \\
\cline { 2 - 4 } & S. aureus & S. epidermidis & E. coli \\
\hline Gentamicina (Control) & $98,5 \pm 0,05$ & $98,3 \pm 0,02$ & $98,9 \pm 0,03$ \\
\hline M. mollis & $92,8 \pm 0,90$ & $90,5 \pm 0,33$ & $91,8 \pm 0,50$ \\
\hline
\end{tabular}

Tabla 4. Concentración mínima inhibitoria (CMI) del aceite esencial frente a tres cepas bacterianas.

\begin{tabular}{|l|c|c|c|}
\hline \multirow{2}{*}{ Aceite esencial } & \multicolumn{3}{|c|}{ Concentración mínima inhibitoria (CMI) } \\
& S. aureus & S. epidermidis & E. coli \\
\cline { 2 - 4 } & 500 & 600 & 500 \\
\hline M. mollis & & \multicolumn{3}{|c|}{. }
\end{tabular}


aromáticas, que contienen $\mathrm{AE}$, como terapia complementaria de las convencionales.

\section{Conclusiones}

Las cepas bacterianas de $S$. aureus, S. epidermidis y $E$. coli, resultaron susceptibles a la acción antibacteriana del AE de M. mollis extraído por el método de hidrodestilación; lo cual, convierte a este AE, cuyo principal constituyente es el agente antimicrobiano carvacrol, como promisorio para el control del componente bacteriano.

\section{Agradecimientos}

Los autores agradecen a las Universidades de Pamplona, Cartagena y Sucre e igualmente al SENA -Centro de Comercio y Servicios, Regional Bolívar-, por facilitar espacio, recursos y tiempo de los investigadores.

\section{Bibliografía}

Baharum SN, Bunawan H, Ghani MaA, Mustapha WAW, Noor NM. Analysis of the chemical composition of the essential oil of POlygonum minus Huds. using two dimensional gas chromatography-time-of-flight mass spectrometry (GC-TOF MS). Molecules. 2010;15(10):7006-7015.

Cano C, Bonilla P, Roque M, Ruiz J. Actividad antimicótica in vitro y metabolitos del aceite esencial de las hojas de Minthostachys mollis (MUÑA): Rev Peru Med Exp Salud Pública. 2008;25(3):298-301.

Carhuapoma M, López S, Roque M, Velapatiño B, Bell C, Whu D. Actividad antibacteriana del aceite esencial de Minthostachys mollis Griseb "Ruyaq Muña". Ciencia e Investigación. 2009;12(2):83-89.

CLSI. Performance standards for Antimicrobial susceptibility testing, $21^{\text {st }}$ international supplements. CLSI Document M100-S21.
Wayne, Pennysylavania, USA: Clinical and Laboratory Standards Institute; 2011.

Gibbons S. Plants as a source of bacterial resistance modulators and antiinfective agents. Phytochem Rev. 2005;4(1):63-78.

Granados C, Yáñez Y, Santafé G. Evaluación de la actividad antioxidante del aceite esencial foliar de Calycolpus moritzianus y Minthostachys mollis de Norte de Santander. Bistua: Revista de la Facultad de Ciencias Básicas. 2012;10(1):12-23.

León G, Osorio MR, Martínez SR. Comparación de dos métodos de extracción del aceite esencial de Citrus Sinensis L. Rev Cuba Farm. 2015;49(4):742-750.

Matiz G, Osorio MR, Camacho F, Atencia M, Herazo J. Diseño y evaluación in vivo de fórmulas para acné basadas en aceites esenciales de naranja (Citrus sinensis), albahaca (Ocimum basilicum L) y ácido acético. Biomédica 2012;32:125-133.

Matiz G, Osorio M, León G. Actividad antibacteriana in vitro de diecinueve aceites esenciales frente a bacterias asociadas al acné, Rev Cubana Farm. 2015;49(1):103-116.

Ramírez A, Stella L, Marín D. Metodologías para evaluar in vitro la actividad antibacteriana de compuestos de origen vegetal. Scientia et Technica. 2009;15(42):263-268.

Tomy GT, Stern GA, Muir DC, Fisk AT, Cymbalisty CD, Westmore JB. Quantifying $\mathrm{C}_{10-\mathrm{C} 13}$ polychloroalkanes in environmental samples by highresolution gas chromatography/electron capture negative ion high-resolution mass spectrometry. Anal Chem. 1997;69(14):2762-71.

Torrenegra M, Granados C, Osorio M, León G. Method comparison of hydrodistillation microwave radiation-assisted (MWHD) front hydrodistillation (HD) in the extraction of essential oil of Minthostachys mollis. Inf Tecnol. 2015;26(1):117-122.

Torrenegra M, Matiz G, León G. Actividad antibacteriana in vitro de aceites esenciales frente a microorganismos implicados en el acné. Rev Cubana Farm. 2015;49(3): 512-523.

Xu J, Zhou F, Ji B, Pei R, Xu N. The antibacterial mechanism of carvacrol and thymol against Escherichia coli. Lett Appl Microbiol. 2008;47(3):174-9. 